\title{
Deceleration of arbitrarily magnetized GRB ejecta: the complete evolution
}

\author{
P. Mimica ${ }^{1}$, D. Giannios ${ }^{2}$, and M. A. Aloy ${ }^{1}$ \\ 1 Departamento de Astronomía y Astrofísica, Universidad de Valencia, 46100 Burjassot, Spain \\ e-mail: Petar.Mimica@uv.es \\ 2 Max Planck Institute for Astrophysics, Box 1317, 85741 Garching, Germany \\ Received 6 August 2008 / Accepted 4 November 2008
}

ABSTRACT

\begin{abstract}
Context. The role of magnetic fields in gamma-ray burst (GRB) flows remains debated. If of sufficient strength, they can leave their signature on the initial phases of the afterglow by substantially changing the backreaction of the flow as a consequence of its interaction with the external medium.

Aims. We attempt to understand quantitatively the dynamical effect and observational signatures of GRB ejecta magnetization on the onset of the afterglow.

Methods. We perform ultrahigh-resolution, one-dimensional, relativistic MHD simulations of the interaction between a radially expanding, magnetized ejecta with the interstellar medium. We require ultrahigh numerical resolution because of the extreme jump conditions in the region of interaction between the ejecta and the circumburst medium. We study the complete evolution of an ultrarelativistic shell to the self-similar asymptotic phase.

Results. Our simulations demonstrate that the complete evolution can be characterized in terms of two parameters, the $\xi$ parameter introduced by Sari and Piran and the magnetization $\sigma_{0}$. We use this fact in producing numerical models in which the shell Lorentz factor $\gamma_{0}$ is between 10 and 20 and rescaling the results to arbitrarily large values of $\gamma_{0}$. We find that the reverse shock is typically weak or absent for ejecta characterized by $\sigma_{0} \gtrsim 1$. The onset of the forward shock emission is strongly dependent on the magnetization. On the other hand, the magnetic energy of the shell is transferred into the external medium on a short timescale (of several times the duration of the burst). The later forward shock emission contains no information about the initial magnetization of the flow. The asymptotic evolution of strongly magnetized shells, after experiencing significant deceleration, resembles that of hydrodynamic shells, i.e. they enter fully into the Blandford-McKee self-similar regime.
\end{abstract}

Key words. gamma rays: bursts - methods: numerical - magnetohydrodynamics (MHD) - shock waves

\section{Introduction}

Gamma ray bursts (GRBs) are understood to be produced by the energy release in an ultrarelativistic outflow. The mechanisms responsible for launching, accelerating the flow and powering the GRB emission are still not well understood. Two alternative sources of the energy content of the flow have been considered. The GRB flow may be dominated initially by thermal energy density creating a fireball (Goodman 1986; Paczynski 1986) or by the energy stored in magnetic fields giving rise to a Poynting-flux dominated flow (PDF, Usov 1992; Thompson 1994; Meszaros \& Rees 1997).

In fireball models, magnetic fields are not dynamically important at any stage of the flow evolution. On the other hand, models of MHD jet acceleration indicate that the conversion of Poynting flux to kinetic energy is partial (Michel 1969; Li et al. 1992; Beskin et al. 1998; Vlahakis \& Königl 2003; Giannios \& Spruit 2006). As a result, the flow remains rather strongly magnetized at large distances where it interacts with the circumburst medium.

The interaction of the fast flow with the external medium is probably responsible the afterglow emission. The initial phases of this interaction depend, to a large extent, on the magnetization of the flow. Strong magnetic fields affect the shock conditions and the internal dynamics of the ejecta. Rees \& Gunn (1974) and Kennel \& Coroniti (1984) presented the first attempts to understand the effect of the magnetization on the shock conditions.
In the ideal MHD limit, they showed that shocks in magnetically dominated flows cannot dissipate energy efficiently. This particular effect was studied recently by examining the shock conditions of the (reverse) shock that propagates into the GRB flow and the resulting emission (Fan et al. 2004; Zhang \& Kobayashi 2005). By accounting for the internal evolution of the magnetized shell, Giannios et al. (2008) argued that even moderately strong magnetic fields can suppress the reverse shock completely and explain the observed paucity of reverse-shock signature in the early afterglow.

We continue previous (semi-)analytical studies of the afterglow phase of magnetized ejecta with relativistic MHD simulations. We follow the deceleration of magnetized ejecta from the initial phases of interaction to the self similar regime in which all energy is transferred to the shocked, external medium. These simulations clarify the dynamical effects of GRB ejecta magnetization and their observational implications for the forward and reverse shock emission. We present a new set of scaling laws that enable us to extrapolate the results of numerical models with moderate values of the initial bulk Lorentz factor $(\sim 15)$ of the ejecta to equivalent models with much larger Lorentz factors $\gtrsim 100$.

\section{Ejecta-medium interaction}

At large distances from the central engine, there is significant interaction between the relativistic ejecta and the external medium. 
This interaction is believed to produce afterglow emission. An important difference between fireballs and PDFs is related to the magnetization of the ejecta at the onset of the afterglow phase. In fireball models, the energy of the flow is dominated by the kinetic energy of baryons at large distances from the central engine. If the flow is launched being Poynting-flux dominated, it is expected to retain a significant fraction of its energy in the form of magnetic energy, the remainder being in kinetic form (Michel 1969; Li et al. 1992; Beskin et al. 1998; Drenkhahn \& Spruit 2002; Lyutikov \& Blandford 2003). Since at least the initial phases of the ejecta-external medium interaction depend on the magnetization of the ejecta (Kennel \& Coroniti 1984; Fan et al. 2004; Zhang \& Kobayashi 2005; Genet et al. 2007), it is possible to differentiate between fireballs and PDF models from afterglow observations. As we show in this work, early afterglow observations are particularly promising in this respect.

\subsection{Previous studies}

The deceleration of non-magnetized ejecta has been studied with both analytical (Rees \& Meszaros 1992; Sari \& Piran 1995) and numerical approaches in one dimension (1D) (Kobayashi et al. 1999) and two dimensions (2D) (Granot et al. 2001; Meliani et al. 2007). The 2D studies are important to follow the latetime lateral spreading of collimated ejecta (Rhoads 1999). On the other hand, the initial phases of ejecta deceleration, in which we are interested, are unaffected by 2D effects and can be studied by assuming a spherically symmetric flow.

Sari \& Piran (1995) considered the case of non-magnetized ejecta assuming a cold shell with (isotropic equivalent) kinetic energy $E$, Lorentz factor $\gamma_{0}$, and width $\Delta_{0}$, which moves against an external medium of density $\rho_{\mathrm{e}}$. This interaction leads to a pair of shocks: one that propagates in the external medium (forward shock) and one that decelerates the ejecta (reverse shock). The strength of the reverse shock depends on the ratio of the density of the shell to the external medium and on the bulk Lorentz factor of the flow. It can be shown that the strength of the reverse shock can be conveniently parametrized by

$\xi \equiv \sqrt{\frac{l}{\Delta_{0}}} \frac{1}{\gamma_{0}^{4 / 3}}$,

where $l=\left(3 E / 4 \pi n_{\mathrm{e}} m_{\mathrm{p}} c^{2}\right)^{1 / 3}$ is the Sedov length, $n_{\mathrm{e}}$ is the number density of electrons in the external medium, $m_{\mathrm{p}}$ is the proton mass, and $E$ is the total energy (kinetic in this case) of the ejecta. In the limit where $\xi \gg 1$, the reverse shock is Newtonian and the shell is said to be "thin". The ejecta do not decelerate much by the time the reverse shock has crossed. If $\xi \ll 1$, we find ourselves in the "thick shell" case, and the reverse shock is relativistic and decelerates the ejecta appreciably (Sari \& Piran $1995)$. For typical parameters of GRB flows, $\xi$ is of the order of unity.

The dynamics of the deceleration of strongly magnetized ejecta have not been studied in similar detail. In addition to the $\xi$ parameter, the ejecta are characterized by the magnetization $\sigma_{0}$ defined to be the ratio of magnetic to kinetic energy in the flow. Kennel \& Coroniti (1984) considered ideal MHD shock conditions in arbitrarily magnetized ejecta with a dominant toroidal field, and showed that the dissipation by the shock becomes weaker as $\sigma_{0}$ increases (e.g. Lyutikov \& Blandford 2003). They applied their analysis to the standing shock of pulsar winds. Zhang \& Kobayashi (2005) focused on the effect of magnetization in the context of GRB afterglows. They ignored the internal evolution of the shell prior to interaction with the external medium and studied the reverse-shock-crossing phase (provided that a reverse shock formed). They found distinct features in the early time light curves because of the magnetization. The shocks from the interaction of the GRB ejecta with the external medium propagate forwards and the shock conditions depend on the distance from the central engine. The Zhang \& Kobayashi (2005) analysis was criticized by Lyutikov (2005) for the assumption about the distance where the ejecta decelerate. Giannios et al. (2008) accounted for the internal evolution of the ejecta and derived an analytic condition for the existence of a reverse shock depending on $\xi$ and $\sigma_{0}$ in a parameter space relevant to GRB flows 1 .

After the reverse shock (if there is one) reaches the back part of the ejecta, there is a transient phase of interaction during which rarefaction waves cross the shocked ejecta and shocked external medium. Gradually most of the energy is transferred into the shocked external medium and the entire structure relaxes to the self-similar blast wave described in Blandford \& McKee (1976). The evolution of the blast wave then depends only on the total energy $E$ and density of the external medium $n_{\mathrm{e}}$ and $n o t$ on $\sigma_{0}$. After the self-similar evolution has been reached, nothing can be inferred about the initial magnetization of the flow.

However, none of these studies have addressed two important questions. First, there is the question of which stage of the interaction a reverse shock forms (if it forms at all). At a short distance from the central engine, the magnetic pressure of the shell is sufficiently high for the shell to rarefy upon interacting with the external medium. This rarefaction may develop into a (reverse) shock at larger distance where the magnetic pressure in the shell drops. Second, although it is clear ignoring radiative losses that the total energy initially in the shell is passed onto the external medium at a distance approximately similar to the Sedov length, which is independent of the magnetization of the flow (Lyutikov 2005), the details of how exactly this occurs depend on the magnetization. These two aspects are closely connected to the energy dissipated in the (forward and reverse) shocks as a function of distance, and consequently, to the afterglow emission from particles accelerated in these shocks. We address this issue by studying the full dynamical interaction from the initial stages to the establishment of the self-similar evolution. To this end, we perform ultra-high-resolution, onedimensional relativistic MHD simulations of shell-medium interaction.

\section{The model for the ejecta}

We focus on the GRB flow at a distance at which there is substantial interaction with the external medium. This interaction probably occurs a considerable time after the acceleration, collimation, and prompt emission phases are completed. After the internal dissipation phase (believed to power the $\gamma$-ray emission) ends, the flow expands radially and cools down. The expansion also produces a dominant toroidal component of the magnetic field. At the so-called Alfvén point, the poloidal $B_{\mathrm{p}}$ and toroidal $B_{\phi}$ field components are expected to be of similar

\footnotetext{
1 While this work was being refereed, Mizuno et al. (2009) published a work addressing the problem of the deceleration of arbitrary magnetized ejecta into an unmagnetized medium, and discussed its implications for GRBs and active galactic nuclei. However, in spite of the undoubtable importance of their studies, the conditions set by these authors (particularly, the use of planar symmetry, and the low density contrast between the magnetized shell and the external medium $\sim 100$ ) are far from those typical of GRB afterglows, especially during the early afterglow propagation.
} 
magnitude. At larger distances, the flux freezing condition corresponds to $B_{\mathrm{p}} \propto 1 / r^{2}$ while the induction equation predicts slower decline for $B_{\phi} \propto 1 / r$. The same scalings would hold if the initial $B$-field was random with $B_{\phi} \gg B_{\mathrm{p}}$ at large distance from the central engine.

We consider radially moving, cold shell of ejecta of width $\Delta_{0}$, total (kinetic and magnetic) energy $E$ that coasts with a bulk Lorentz factor $\gamma_{0}$. The magnetic content of the flow is parametrized with the magnetization parameter $\sigma_{0}$, which represents the magnetic-to-kinetic energy ratio in the shell. The flow is assumed to move with super-fast magnetosonic speeds (i.e. $\gamma_{0}^{2}>1+\sigma_{0}$; for studies of the opposite limit, see Lyutikov 2006; and Genet et al. 2007). For the simulations presented below, the shell is located at an initial distance $r_{0}$ from the central engine. The choice of $r_{0}$ is important, since $r_{0}$ should be sufficiently small not to affect the subsequent interaction of the ejecta with the external medium. We must set $r_{0}$ to be smaller than any of the characteristic radii that appear when considering the deceleration of magnetized ejecta. These radii are the "contact" radius and the "reverse-shock crossing" radius to be defined in the next section.

\subsection{Characteristic distances}

In the super-fast magnetosonic flow under consideration, the various parts along the radial direction drop out of MHD contact during the acceleration phase. It can be shown that for a cold flow that coasts with constant speed with dominant toroidal field the magnetization remains constant. The time it takes for a fast MHD wave to cross the width of the shell is therefore fixed. The expansion timescale $t_{\exp }=r / \gamma_{0} c$ is initially much shorter than that of MHD waves but increases linearly with distance from the central engine. At the so-called "contact" radius $r_{\mathrm{c}}$, MHD waves cross the width of the shell on a timescale comparable to the expansion timescale (Giannios et al. 2008)

$r_{\mathrm{c}} \simeq \Delta_{0} \gamma_{0}^{2}\left(\sqrt{\frac{1+\sigma_{0}}{\sigma_{0}}}-1\right)$.

After contact is established, the shell is no longer in pure ballistic motion and internal evolution because MHD forces can no longer be ignored. On the other hand, in non-magnetized ejecta the sound speed declines sharply with distance due to adiabatic expansion, and the motion is unaffected by the pressure of the shell.

A second important radius is that at which the reverse shock reaches the rear part of the ejecta. This radius can be identified using the formulation of Zhang \& Kobayashi (2005) by solving the ideal MHD shock conditions for arbitrarily magnetized ejecta (see also Fan et al. 2004, for the case of mildly magnetized ejecta). Their analysis describes the reverse-shock crossing-phase provided that there is a reverse-shock forming. The reverse-shock crossing radius can be expressed approximately as (Giannios et al. 2008)

$r_{\mathrm{rs}} \simeq l^{3 / 4} \Delta_{0}^{1 / 4} / \sqrt{1+\sigma_{0}}$.

The Zhang \& Kobayashi (2005) analysis does not take into account the internal evolution of the shell. It is thus accurate when such evolution insignificant, i.e. when $r_{\mathrm{rs}}<r_{\mathrm{c}}$.

The initial distance $r_{0}$ where the shell is set up up must be $r_{0} \ll \min \left[r_{\mathrm{c}}, r_{\mathrm{rs}}\right]$ so that the simulation begins sufficiently early to follow both any rarefaction waves within the shell, and shocks caused by the interaction with the external medium.

\subsection{Characteristic quantities}

In this paper, we frequently use the following definition of the normalized time of observation for a model with parameters $\gamma_{0}$, $\Delta_{0}$, and $r_{0}$ :

$t_{\mathrm{obs}}:=\Delta_{0}^{-1}[c t-r]$,

where $t_{\text {obs }}$ is the time for observation of a signal transmitted from radius $r$ at time $t$ in the GRB frame or laboratory frame, normalized to the light-crossing time of the initial width of the shell $\Delta_{0} / c$. As we show in Sect. 4.4.3, this definition of $t_{\text {obs }}$ enables us to compare properties (in the observer frame) of shells with the same value of $\xi$, independent of their initial Lorentz factor.

We also often base our arguments on the relative Lorentz factor $\gamma_{\text {rel }}$ between two parts of the fluid separated by the shock front. For ultrarelativistic flows, we use

$\gamma_{\mathrm{rel}}:=\frac{1}{2}\left(\frac{\gamma_{\mathrm{a}}}{\gamma_{\mathrm{b}}}+\frac{\gamma_{\mathrm{b}}}{\gamma_{\mathrm{a}}}\right)$

where $\gamma_{\mathrm{a}}$ and $\gamma_{\mathrm{b}}$ are the Lorentz factors of the fluid ahead and behind of the shock, respectively. We point out that $\gamma_{\text {rel }}$ depends only on the ratio $\gamma_{\mathrm{a}} / \gamma_{\mathrm{b}}$.

\subsection{Numerical models}

Although the problem is characterized by several parameters $E$, $n_{\mathrm{e}}, \Delta_{0}, \gamma_{0}$, and $\sigma_{0}$, to complete a systematic study of the shellmedium interaction, we need to focus on the combination of the first four parameters that are given by

$\xi \propto\left(E / n_{\mathrm{e}}\right)^{1 / 6} / \Delta_{0}^{1 / 2} \gamma_{0}^{4 / 3}$

and $\sigma_{0}$. We demonstrate and quantify this statement in the next Section. To simplify the analysis, we restrict ourselves to the case in which the external medium density is uniform $\left(\rho_{\mathrm{e}} \approx 3 \times\right.$ $10^{-4} \rho_{0}\left(1+\sigma_{0}\right) ; \rho_{0}$ being the initial shell density, and leave the study of stratified external media to future work.

As in the case of unmagnetized ejecta, we use the Sari-Piran parameter $\xi$ to characterize partly the strength of the reverse shock. In the magnetized case, the shock strength is certainly not uniquely constrained by $\xi$. An additional parameter, $\sigma_{0}$, must be introduced to describe fully the reverse-shock strength of arbitrarily magnetized flows. Thus, one deals with a $\xi-\sigma_{0}$ plane in exploring different cases for the initial phases of shell-external medium interaction. Here, we explore the $\xi \sim 1$ regime that is relevant to typical GRB parameters. Numerical reasons limit us to the $0 \leq \sigma_{0} \leq 3$ range of the magnetization parameter.

Our different models are summarized in Table 1 . The $\xi=$ 1.1 models (thin shells) are characterized by $E=3.33 \times 10^{53} \mathrm{erg}$, $\Delta_{0}=10^{15} \mathrm{~cm}, \gamma_{0}=15, n_{\mathrm{e}}=10 \mathrm{~cm}^{-3}$. The $\xi=0.5$ models (thick shells) have a factor of ten higher total energy $E$ and the shell width $\Delta_{0}$, while $\gamma_{0}$ and $n_{\mathrm{e}}$ remain fixed. The "continuous flow" model (to be discussed in more detail in Sect. 4.3) describes a flow of constant total (kinetic + Poynting) luminosity of $L=10^{49} \mathrm{erg} / \mathrm{s}$ that moves with $\gamma_{0}=15$ and collides with an external medium of number density $n_{\mathrm{e}}=10 \mathrm{~cm}^{-3}$. In all models, $r_{0}=5 \times 10^{16} \mathrm{~cm}$.

One may notice that the models are characterized by an unrealistically low Lorentz factor $\gamma_{0}=15$ and wide shells $\Delta_{0} \sim 10^{15}$ $\mathrm{cm}$ with respect to that expected for a GRB flow (i.e. $\gamma_{0} \gtrsim 100$, $\left.\Delta_{0} \lesssim 3 \times 10^{12} \mathrm{~cm}\right)$. These parameters were chosen so that the problem was resolved reliably with our RMHD code. While models with $\gamma_{0} \gtrsim 100$ in combination with extreme density and 
Table 1. Parameters of the numerical models.

\begin{tabular}{llll}
\hline \hline$\sigma_{0}$ & 0 & 1 & 3 \\
\hline Thin shell $(\xi=1.1)$ & $\sqrt{ }$ & $\sqrt{ }$ & \\
Thick shell $(\xi=0.5)$ & $\sqrt{ }$ & $\sqrt{ }$ & \\
Continuous flow & & $\sqrt{ }$ & $\sqrt{ }$ \\
\hline
\end{tabular}

magnetic field jumps at the edge of the ejecta shell are not feasible at this stage, we propose a method to extrapolate the results of the $\gamma_{0}=15$ simulation by rescaling appropriately the initial conditions. Furthermore, we completed simulations with $\gamma_{0}=10,20$, where we demonstrate the accuracy of the rescaling procedure (see Sect. 4.4).

\section{Results}

To derive the results presented in this Section, we solve the equations of RMHD in 1D spherical geometry with a magnetic field perpendicular to the direction of propagation of the fluid, i.e., with a purely toroidal magnetic field. The system of RMHD equations, and the numerical tests we have completed to identify the appropriate numerical resolution for our experiments (between $10^{4}$ and $6 \times 10^{4}$ cells to resolve the initial radial width of the ejecta) are shown in Appendix A. Finally, all our models have were executed until the bulk Lorentz factor behind the forward shock had declined to $\gamma \sim 2-3$. By that time, the shell had experienced a substantial deceleration and fully entered into the Blandford-MacKee self-similar regime.

\subsection{Non-magnetized shells}

The $\sigma_{0}=0$ models exhibit the well known features expected from analytical results (Sari \& Piran 1995) and simulations (Kobayashi et al. 1999). The thin shell $(\xi=1.1)$ model is characterized by a Newtonian-to-mildly-relativistic reverse shock. The reverse shock crosses the shell at a distance $r_{\mathrm{rs}}=3 \times 10^{17} \mathrm{~cm}$, which agrees to within $\sim 10 \%$ with the analytical estimate from Eq. (3). At this distance, the relative Lorentz factor of the shocked ejecta with respect to the unshocked shell is $\gamma_{\text {rel }} \simeq 1.18$. The thick shell $(\xi=0.5)$ model finds itself closer to the "relativistic reverse shock" regime with $r_{\mathrm{rs}}=9.5 \times 10^{17} \mathrm{~cm}$ (to within $\sim 10 \%$ of the initial estimate) and $\gamma_{\text {rel }} \simeq 1.60$. In both runs, after the reverse shock crosses the shell, a rarefaction begins at the rear part of the shell and propagates forwards (a similar rarefaction happens in the magnetized case, which we label $R_{2}$ in Fig. 1). The rarefaction crosses the contact discontinuity, generated in the leading radial edge of the shell (an equivalent contact-discontinuity arises in the magnetized case: see "CD" in Fig. 1), and reaches the forward shock as the shell approaches $r \sim 1.6 r_{\mathrm{rs}} \simeq 5 \times 10^{17} \mathrm{~cm}$ and $r \sim 1.6 r_{\mathrm{rs}} \simeq 1.6 \times 10^{18} \mathrm{~cm}$, in the thin- and in the thick-shell case, respectively. At this stage, $\sim 90 \%$ of the energy of the shell has been transferred into the shocked external medium. Within a factor of $\sim 2$ in radius, the blastwave fully relaxes to the Blandford-McKee self-similar solution.

The different components of the total energy (see e.g., Mimica et al. 2007) as function of radius of the front shock (FS) are shown in Fig. 2. For the thin-shell model, the peak of the thermal energy of the shell (approximately 9\% of the total energy contained in the ejecta) identifies the position of the reverse shock (RS) crossing of the shell. Beyond $5 \times 10^{17} \mathrm{~cm}$, most of the energy that was initially in the shell is transferred into the shocked external medium. The apparent "disappearance" of the

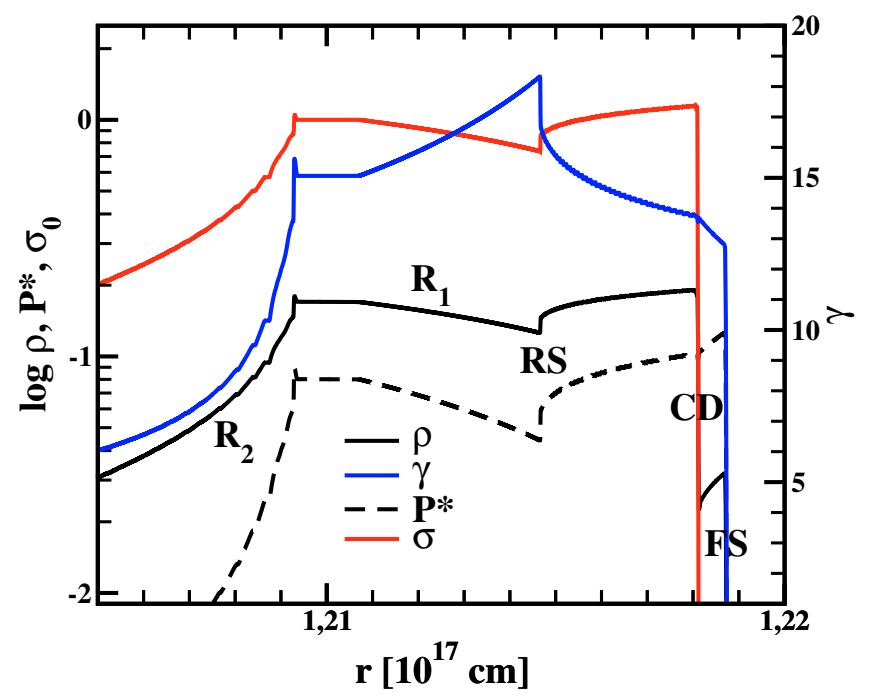

Fig. 1. Snapshot of the thin magnetized shell evolution taken after the RS has formed and before it has crossed the shell. Full and dashed black lines show the logarithms of the rest-mass density (normalized to the initial shell density $\rho_{0}$ ) and of the pressure (normalized to $\rho_{0} c^{2}$ ). The red line shows the logarithm of the magnetization $\sigma$, while the blue line shows the fluid Lorentz factor $\gamma$ in the linear scale. All quantities are shown as a function of radius $r$. Positions of the forward shock (FS), contact discontinuity (CD), reverse shock (RS), and left- $\left(R_{1}\right)$ and rightgoing $\left(R_{2}\right)$ rarefactions have been indicated. Another rarefaction exists (moving backwards in the external medium) and another contact discontinuity that forms at the rear edge of the shell, which are not shown here. Both structures are located to the right of $R_{2}$. The unshocked external medium is located in front of the FS and its density $\rho_{\mathrm{e}} \approx 6 \times 10^{-4} \rho_{0}$ is lower than the minimum density shown in the plot. The rarefaction $R_{2}$, the CD and the FS display a qualitatively similar profile in the nonmagnetized case. The rarefaction $R_{1}$ and the late steepening of the conditions at its tail, resulting into the formation of RS, are specific of magnetized ejecta.

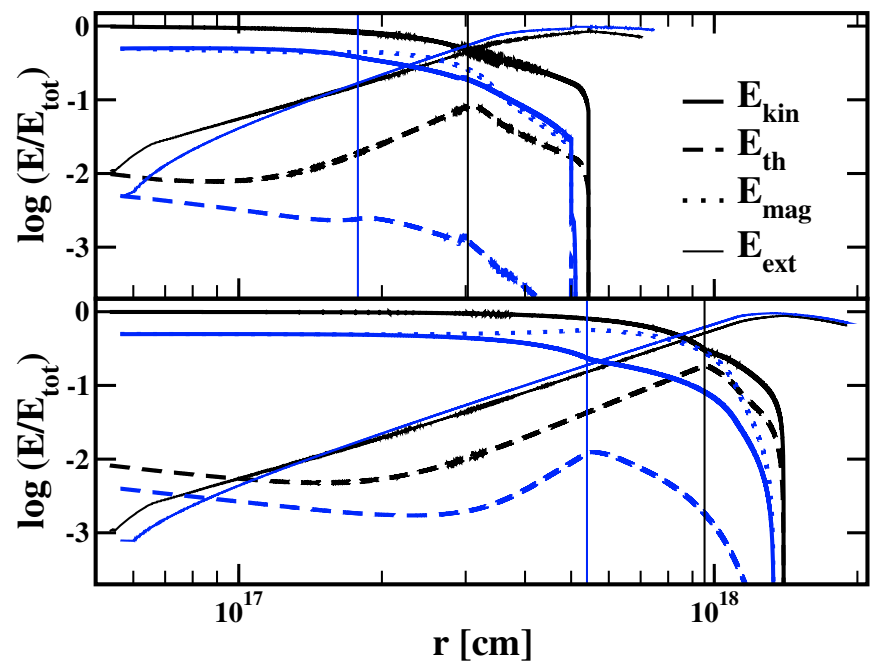

Fig. 2. Energy in different components (normalized to the initial total energy in the shell) as a function of the radius of the FS. Upper and lower panels show the thin $(\xi=1.1)$ and thick $(\xi=0.5)$ shell models, respectively. Black and blue lines correspond to un-magnetized $(\sigma=0)$ and magnetized $(\sigma=1)$ models, respectively. Thick full, dashed, and dotted lines indicate the kinetic, thermal, and magnetic energy of the shell, respectively. The thin full line shows the total energy in the shocked external medium. Thick vertical lines denote the radius of the FS at the moment when the RS has crossed the shell. 


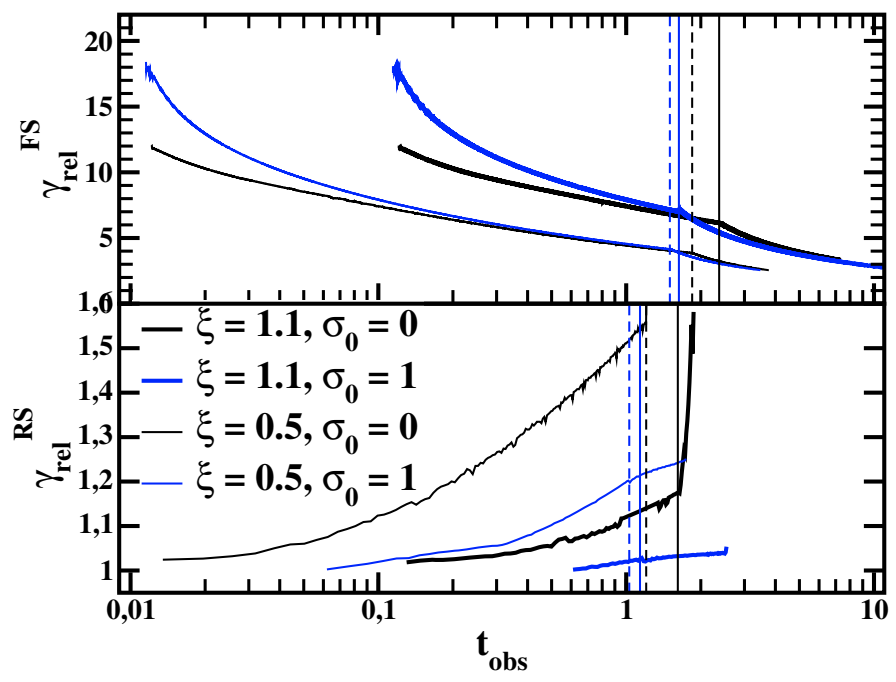

Fig. 3. Relative Lorentz factor at the FS (upper panel) and RS (lower panel) as a function of the normalized observer time $t_{\mathrm{obs}}$ (Eq. (4)). Full and dashed lines correspond to the thin and thick shell models, respectively. Black and blue colors denote non-magnetized and magnetized models, respectively. The vertical lines in the lower panels denote the time of observation when the RS crosses the shell. In the upper panel, the vertical lines denote the time of observation when the rarefaction $R_{2}$, which originates in the rear edge of the shell and interacts with the RS, catches up with the FS.

shell at $r \approx 5.4 \times 10^{17} \mathrm{~cm}$ is a numerical artifact of the grid remapping ${ }^{2}$. However, this effect is irrelevant to the discussion of the features in which we are interested, since all occur before the shell "disappears", both in non-magnetized and magnetized models. For the unmagnetized thick-shell runs, we see that the reverse shock dissipates more energy from the shell, reaching approximately $18 \%$ of the total shell energy by the time it crosses the shell.

In Fig. 3, we show the relative Lorentz factor at the FS and the RS as a function of observer time. Both shocks form immediately after allowing the initial shell to evolve. The RS becomes stronger with distance, as evident in the increase of $\gamma_{\mathrm{rel}}$ with $t_{\mathrm{obs}}$. This strengthening continues until it reaches the rear part of the shell, where it encounters a far less dense medium, resulting in a kink in the RS curves (marked with vertical black lines in Fig. 3). The peak of the emission associated with the RS is expected to occur at the moment the RS breaks out of the shell, since afterwards the density, pressure, and velocity of the shocked medium decrease abruptly, and precisely there it reaches its maximum strength. For the thin-shell case, this happens slightly after the burst, i.e. at observer time $t_{\mathrm{obs}} \sim 1$.6. For the thick shell, the peak of the RS emission appears at the end of the burst, at a time $t_{\text {obs }} \sim 1.2$.

The Lorentz factor of the external medium just behind the FS declines as a function of radius. An interesting feature is the change in the slope of $\gamma_{\text {rel }}^{\mathrm{FS}}\left(t_{\mathrm{obs}}\right)$ at time $t_{\mathrm{obs}}=2.5$ for the thin shell (at time $t_{\mathrm{obs}}=1.84$ for the thick shell), which is a result of the rarefaction $R_{2}$ (Fig. 1) reaching the forward shock. For a short transient period, the FS reduces its strength because the density behind it is reduced by the action of the rarefaction $R_{2}$. The decay of $\gamma_{\text {rel }}^{\mathrm{FS}}\left(t_{\mathrm{obs}}\right)$ is more rapid for a time interval $\Delta t_{\mathrm{obs}} \sim 0.5$ after

\footnotetext{
2 As described by Mimica et al. (2007), grid remapping enables us to follow the evolution of a localized shell over large distance by repeated remapping of the numerical grid. In this work the grid always follows the front shock, so that, once the shell has been slowed down by the reverse shock, it is eventually "lost" from the grid.
}

the rarefaction $R_{2}$ catches up with the FS. Thus, we expect the early afterglow emission to weaken transiently more rapidly with time. Later, the time dependence of $\gamma_{\mathrm{rel}}^{\mathrm{FS}} \propto t_{\mathrm{obs}}^{-3 / 8}$ expected from the self-similar solution, is gradually established.

\subsection{Magnetized shells}

The initial phases of deceleration of strongly magnetized ejecta distinctly differ from those that are unmagnetized in a way related to the magnetic pressure of the ejecta. As discussed in Giannios et al. (2008), the discontinuity in the physical conditions between the shell and the external medium results in the development of two Riemann structures, each every radial (rear and forward) edge of the shell. The waves into which each of these Riemann structures decompose are combinations of shocks and/or rarefactions separated by contact discontinuities. If the magnetic pressure is sufficiently high, instead of a typical double-shock structure, a right-going shock (FS) and a left-going rarefaction $\left(R_{1}\right)$ develop from the forward edge of the shell (Fig. 1). Similarly, at the backward edge of the shell, a rarefaction (not shown in Fig. 1) moving backwards (in the shell comoving-frame) into the external medium develops, as well as a rarefaction that penetrates the shell.

The expansion of the shell produces a decrease in the magnetic pressure and the Lorentz factor of contact until the "transition" radius, $r_{\mathrm{tr}}$, is reached, where $\gamma_{\mathrm{CD}}=\gamma_{0}$. This distance can be estimated by balancing between the pressure of the shocked external medium $P=4 \gamma_{0}^{2} n_{e} m_{p} c^{2} / 3$ and the magnetic pressure of the shell $P_{\mathrm{B}}=B^{2} / \gamma_{0}^{2} 8 \pi=E \sigma_{0} / 8 \pi r^{2} \gamma_{0}^{2} \Delta_{0}\left(1+\sigma_{0}\right)$, and solving for the radius

$r_{\mathrm{tr}}=\left(\frac{3 E \sigma_{0}}{32 \pi n_{\mathrm{e}} m_{\mathrm{p}} c^{2} \gamma_{0}^{4} \Delta_{0}\left(1+\sigma_{0}\right)}\right)^{1 / 2}$

From this distance onwards, the shell decelerates with respect to its initial $\gamma_{0}$. Some time after the rarefaction $R_{1}$ has propagated backwards into the shell, a new shock develops inside the rarefaction fan due to the radial expansion of the shell (RS in Fig. 1). This shock sweeps backwards through the shell and, therefore, is effectively a reverse shock. Remarkably, this shock does not immediately originate in the initial discontinuity at the leading radial edge of the shell. It develops at the more rapidly moving parts of the rarefaction fan and not directly at the contact discontinuity separating the FS and $R_{1}$ (see Fig. 1). The reason is that $\sigma \propto r^{2} \rho$ in the cold, magnetized shell (see Appendix A), and therefore it decreases in the rarefaction fan. The formation of this shock can only occur when the initial shell expands spherically, and not if the shell is assumed to evolve under conditions of planar (Cartesian) symmetry (as assumed by Mizuno et al. 2009). The fact that $\sigma$ decreases in the rarefaction and the rarefaction profile steepens due to the spherical geometry, leads to a decrease of the fast magnetosonic speed across the entire rarefaction fan. The decrease is larger right at the head of the rarefaction where, eventually, a shock forms ${ }^{3}$. We therefore realize that the structure of the flow is far more complex than in the non-magnetized case, since it has developed a RS inside the rarefaction fan of $R_{1}$.

The shock is initially weak $\left(\gamma_{\text {rel }}^{\mathrm{RS}} \simeq 1\right)$ and remains so during the period in which it sweeps up the entire (thin) shell (Fig. 3). When it reaches the back edge of the shell, $\gamma_{\text {rel }} \simeq 1.03$, i.e., it remains Newtonian. The reverse shock dissipates a negligible amount of energy $(\sim 0.1 \%$ of the total energy in the shell).

\footnotetext{
3 We thank the referee for pointing out that, without this decrease of the fast speed, a shock would form either immediately or never at all.
} 
It becomes stronger when a thick shell is considered, reaching $\gamma_{\mathrm{rel}} \simeq 1.2$ by the time it reaches the rear radial edge of the shell (Fig. 3, lower panel). Thus, this RS sweeping a thick shell leads to a higher dissipation of energy ( $\sim 1 \%$ of the total). However, the dissipated energy is still a factor of $\sim 20$ lower than in the non-magnetized thick-shell model. In the thin-shell case, the local maximum of the thermal energy at $1.7 \times 10^{17} \mathrm{~cm}$ (Fig. 2 upper panel) corresponds to the RS crossing. Afterwards, most of the energy concentrates into the shocked external medium at distance $r>4 \times 10^{17} \mathrm{~cm}$, when the evolution becomes similar to that of the unmagnetized shell.

At early times, the Lorentz factor of the medium just behind the forward shock is higher than that of the shell, due to the initial rarefaction. This initially leads to $\gamma_{\mathrm{rel}}>15$ until the "transition" radius is reached (Fig. 3). The fact that initially $\gamma_{\text {rel }}>\gamma_{0}$ is a unique feature of magnetized ejecta (in unmagnetized ejecta $\gamma<\gamma_{0}$ always). This initial phase also appears in the early afterglow of the electromagnetic model (Lyutikov 2006; Genet et al. 2007).

The magnetization affects the (reverse) shock conditions and, as a result, the reverse shock crosses a magnetized shell more rapidly than an unmagnetized one (the vertical blue lines appear to the left of the vertical black lines in the lower panel of Fig. 3). This feature was revealed in the study of Fan et al. (2004). Our simulations demonstrate that the rarefaction $R_{2}$ also crosses (in observer time) a magnetized more rapidly shell than a unmagnetized one.

On a timescale of a few (thick shell) or several (thin shell) times that of the duration of the burst, the $\sigma=0$ and $\sigma=1$ models exhibit similar evolution. At this stage, almost all the shell energy has been transferred to the shocked external medium (Fig. 2). The two models have the same total energy and relax to identical asymptotic self-similar solutions (note the similarity between the rising parts of the solid thin blue and black lines in Fig. 2). The forward shock emission beyond this time reveals nothing about the initial magnetization. We note, however, that there is a prolonged RS crossing phase at the tail of the magnetized ejecta, and some residual energy remains in the form of Poynting flux at later times that may power some (energetically weak) afterglow features.

\subsection{Dissipation by the reverse shock}

Our simulations can quantitatively answer the question of how much energy is dissipated when the reverse shock propagates into ejecta of different magnetization $\sigma_{0}$ and parameter $\xi$. For practical reasons, the simulations were limited to a few models ${ }^{4}$. On the other hand, they can be used in evaluating the accuracy and limitations of previous (semi-)analytical studies (Fan et al. 2004; Zhang \& Kobayashi 2005; Giannios et al. 2008) and exploring a larger parameter space of $\xi$ and $\sigma_{0}$.

The "continuous flow" models with $\sigma_{0}=1$ and $\sigma_{0}=3$ describe spherical flows of constant (as functions of radius) luminosity $L$, magnetization $\sigma_{0}$, and Lorentz factor $\gamma_{0}$ that collide with a uniform external medium of number density $n_{\mathrm{e}}$. Initially, the interface between the two media is set to be at some distance

\footnotetext{
${ }^{4}$ Each simulation takes between 50 and 200 thousand hours of computer time using between 32 and 320 processors (depending whether we compute thin, thick, or continuous flow models) on Mare Nostrum (http://www.bsc.es/plantillaA.php?cat_id=5). The typical external storage requirements of one model vary between 10 (thin) and 100 (thick models) gigabytes, since relatively frequent output of the fluid state is needed to obtain a satisfactory coverage of the fluid evolution needed for the post-processing calculations.
}

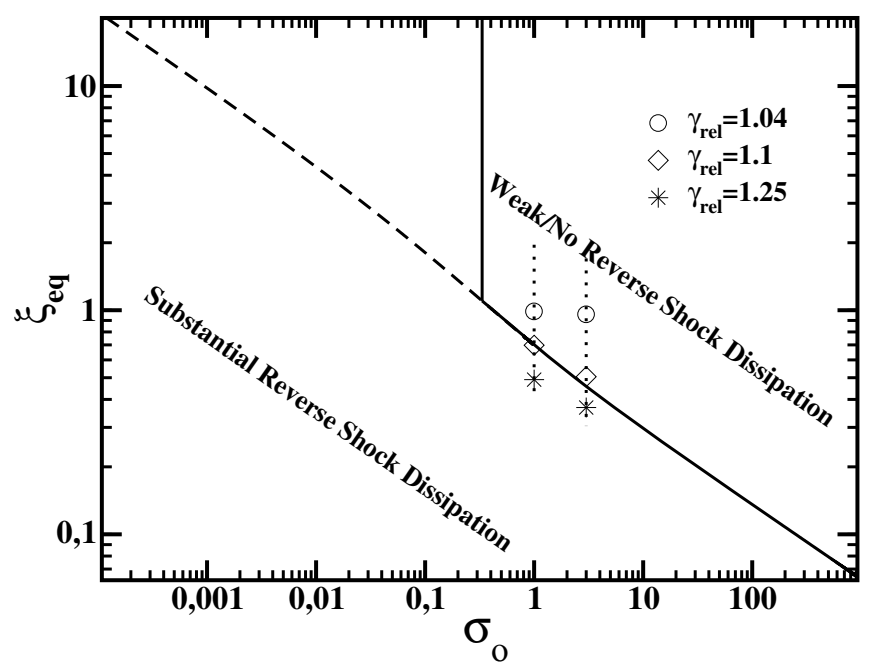

Fig. 4. Energy dissipation in the reverse shock in $\xi-\sigma_{0}$ parameter space. The circles, diamonds, and asterisks indicate the equivalent $\xi$ of the shell for which the relative Lorentz factor $\gamma_{\text {rel }}$ of the shocked ejecta with respect to the unshocked ejecta becomes $1.04,1.1$, and 1.25 respectively. The dotted curves show the region probed by the $\sigma_{0}=1$ and $\sigma_{0}=3$ simulations. The solid line marks the "no-reverse-shock boundary" as estimated by Giannios et al. (2008). In the "weak/no-reverseshock dissipation" region, the shock converts less than $\sim 0.3 \%$ of the total energy of the shell into heat.

$r_{0}$ (see Sect. 3 for the choice of $r_{0}$ ) and the system is allowed to evolve. With these models, we can focus on the interface of interaction between the shell and external medium and, therefore, track in significant detail the formation and strengthening of the reverse shock with time (or equivalently radius). This configuration allows us to follow the strength of the reverse shock for different "equivalent thickness" of shells in a single simulation.The idea behind the equivalent thickness is to measure the penetration distance from the contact discontinuity to the reverse shock, and assume a shell whose initial thickness $\Delta_{0}$ is equal to this distance. We can then use Eq. (1) to obtain the equivalent $\xi$ of the assumed shell. The consequence of this is that the more the reverse shock penetrates the flow, the thicker equivalent shell it probes for the fixed magnetization $\sigma_{0}$. We define equivalent $\xi$ (taking into account that $E \simeq L \Delta_{0} / c$ )

$\xi_{\mathrm{eq}}:=\left(\frac{3 L}{4 \pi n_{\mathrm{e}} m_{\mathrm{p}} c^{3}}\right)^{1 / 6} \Delta_{0}^{-1 / 3} \gamma_{0}^{-4 / 3}$

for which it is evident that a thicker equivalent shell corresponds to a lower $\xi$. A continuous flow model probes a line of constant $\sigma_{0}$ in the $\xi-\sigma_{0}$ plane, as shown in Fig. 4.

A convenient measure of the strength of the reverse shock is the relative Lorentz factor $\gamma_{\text {rel }}$ of the unshocked ejecta with respect to the shocked ejecta (Eq. (5)). In Fig. 4, we mark the locations where the $\gamma_{\text {rel }}$ becomes $1.04,1.1$, and 1.25. We have argued in (Giannios et al. 2008) that for $r_{\mathrm{rs}} \ll r_{\mathrm{c}}$, the magnetization of the flow cannot prevent the formation of a shock in the ejecta and vice versa. The curve defined by setting $r_{\mathrm{rs}}=r_{\mathrm{c}}$ (thick black line in Fig. 4) can thus be used as a proxy to mark the region where a reverse shock forms.

As one can see in Fig. 4, the region in which a reverse shock exists is larger than that estimated by (Giannios et al. 2008). This is due to the late RS formation that has been revealed by the numerical simulations. This effect was unaccounted for in our previous analytic estimates. However, the analytic prediction that the reverse shock emission from models whose 
parameters are in the region $r_{\mathrm{rs}} \sim r_{\mathrm{c}}$ would not be observed is still qualitatively valid, since the dissipation from the shock is weak. On the solid line, $\gamma_{\text {rel }} \sim 1.1$ and becomes higher only for lower values of $\xi$. For $\gamma_{\text {rel }} \sim 1.1$, the shock converts only a fraction of $\sim f_{\mathrm{b}}\left(\gamma_{\mathrm{rel}}-1\right) /\left(1+\sigma_{0}\right) \ll 0.1$ of the energy of the shell into heat. Here $f_{\mathrm{b}} \sim 0.3$ for $\sigma_{0} \sim 1$ (see e.g., Zhang \& Kobayashi 2005). Integrating the thermal energy in the shocked shell for the snapshot for which $\gamma_{\mathrm{rel}}=1.1$, we find that it accounts only for $\sim 0.3 \%$ and $\sim 0.1 \%$ of the total energy of the shocked shell in the $\sigma_{0}=1$ and $\sigma_{0}=3$ models respectively. This reveals that a shock, though there, still dissipates weakly "above" the solid line of Fig. 4.

\subsection{Rescaling of the results to arbitrarily high $\gamma_{0}$}

Our simulations correspond to an ultrarelativistic shell of material interacting with the external medium that has the qualitative characteristics expected at the onset of the afterglow phase of the GRB ejecta. Nevertheless, they have two parameters that are unrealistic with respect to that expected in a GRB, namely the initial bulk Lorentz factor $\gamma_{0}$ and the thickness of the shell $\Delta_{0}$. GRBs are believed to originate from flows with $\gamma_{0} \gtrsim 100$, while the flow thickness is probably connected to the observed duration of the burst by $\Delta_{0} \sim c t_{\mathrm{GRB}} \lesssim 10^{13} \mathrm{~cm}$. However, numerical reasons compel us to simulate shells which have $\gamma_{0} \sim 15$ and $\Delta_{0} \sim 10^{15}-10^{16} \mathrm{~cm}$. In this section we present a method in which our results can reliably be rescaled to GRB-relevant parameters.

\subsubsection{Motivation for the proposed rescaling}

We first focus on unmagnetized GRB ejecta and then extend our discussion to include magnetization. For $\sigma_{0}=0$, the problem of the interaction between an ultrarelativistic and thin cold shell with an external medium is defined by four parameters $E, \Delta_{0}$, $\gamma_{0}$, and $n_{\mathrm{e}}$. The evolution of this configuration does not depend on the individual parameters but a specific combination of them can be expressed by $\xi \propto\left(E / n_{\mathrm{e}}\right)^{1 / 6} / \Delta_{0}^{1 / 2} \gamma_{0}^{4 / 3}$. The parameter $\xi$ determines, for example, the relative Lorentz factor $\gamma_{\text {rel }}$ of the reverse shock (Sari \& Piran 1995). For the ultrarelativistic flow under consideration, $\gamma_{\text {rel }} \simeq F\left(\gamma_{\mathrm{sh}} / \gamma_{0}\right)$ (see Eq. (5)), where $\gamma_{\mathrm{sh}}$ represents the Lorentz factor of the shocked ejecta when the shock reaches their rear part. For fixed $\xi$, this means that $\gamma_{\mathrm{sh}}$ is a fixed fraction of $\gamma_{0}$ independently of the value of $\gamma_{0}$. For example, since in our $\left(\sigma_{0}, \xi\right)=(0,0.5)$ model we found that $\gamma_{\mathrm{sh}} \simeq 0.35 \gamma_{0} \simeq 5.2$, one can predict that a non-magnetized shell with $\xi=0.5$ and arbitrary $\gamma_{0} \gg 1$ is characterized by $\gamma_{\mathrm{sh}} \simeq 0.35 \gamma_{0}$ at the moment of the RS crossing.

The idea behind rescaling is to solve (numerically) the evolution of a shell with $\gamma_{0,1}$ and $\Delta_{0,1}$, and then predict (without simulating) the evolution of a second shell with $\gamma_{0,2}$ (usually higher than $\left.\gamma_{0,1}\right)$ and $\Delta_{0,2}=\Delta_{0,1}\left(\gamma_{0,2} / \gamma_{0,1}\right)^{-8 / 3}$ which has the same $\xi^{5}$. The distance from the central engine at which the reverse shock crosses the second shell is $r_{\mathrm{rs}, 2}=r_{\mathrm{rs}, 1}\left(\gamma_{0,1} / \gamma_{0,2}\right)^{2 / 3}$ (see Eq. (3)). The same relation connects the distances given by $r_{\gamma_{0}} \simeq l \gamma_{0}^{-2 / 3}$ (see Sari \& Piran 1995) where the shells " 1 " and " 2 " enter the deceleration phase after accumulating a fraction $M_{0} / \gamma_{0}$ of their own initial mass $M_{0}$. This indicates that the characteristic distances of the shell-medium interaction for the shell " 2 " are shifted by a factor $\left(\gamma_{0,1} / \gamma_{0,2}\right)^{2 / 3}$ with respect to those of the

\footnotetext{
${ }^{5}$ For the simplicity of the discussion, we fix $E$ and $n_{\mathrm{e}}$ for the two shells. We generalize our expressions to arbitrary values of $E$ and $n_{\mathrm{e}}$ in the Appendix B.
}

shell "1". We postulate that the same is true throughout the evolution of the system. More precisely, we claim that by rescaling the Lorentz factor from $\gamma_{0,1}$ to $\gamma_{0,2}$ one can predict the evolution of a shell " 2 " by using that of the shell " 1 " providing that one also rescales the distance to $r^{\prime} \rightarrow r\left(\gamma_{0}^{\prime} / \gamma_{0}\right)^{2 / 3}$.

We further extend the previous postulate by adding up the possibility that the shell was magnetized, i.e., we extend the previous claim to the case $\sigma_{0} \geq 0$. The evolution of a thin, ultrarelativistic shell of thickness

$\Delta_{0,2}=\Delta_{0,1}\left(\gamma_{0,2} / \gamma_{0,1}\right)^{-8 / 3}$

is self-similar to that of a shell with the same $\xi$ and $\sigma_{0}$, and width $\Delta_{0,1}$.

\subsubsection{Mathematical description of the rescaling}

Here, we provide the expressions for transformation of the solutions for models with the same total energy $E$ and density of the external medium $n_{\mathrm{e}}$. On more mathematical terms, the postulated recipe for transforming from one solution to another is the following. Suppose the bulk Lorentz factor $\gamma_{1}\left(r_{1}, t_{1}\right)$ of the shell " 1 " at is known $\left(t_{1}\right.$ is the time in the rest frame of the GRB engine or laboratory frame), and we define the quantity $f:=\gamma_{0,2} / \gamma_{0,1}$. We further assume that the bulk Lorentz factors of both shells at any time other than the initial one are linked by

$\gamma_{2}\left(r_{2}, t_{2}\right)=f \gamma_{1}\left(r_{1}, t_{1}\right)$

where

$t_{2}=f^{-2 / 3} t_{1}$

$r_{2}=r_{\mathrm{FS}, 1}\left(t_{1}\right) f^{-2 / 3}+\left(r_{1}-r_{\mathrm{FS}, 1}\left(t_{1}\right)\right) f^{-8 / 3}$.

Here $r_{\mathrm{FS}, 1}\left(t_{1}\right)\left(r_{\mathrm{FS}, 2}\left(t_{2}\right)\right)$ represents the radius of the forward shock of the shell "1" ("2") as function of time. The other physical quantities in shell "2" can be derived from $\gamma_{2}$ by using standard expressions for the forward-shock conditions. The (gas or magnetic) pressure $P$ in both the shell and shocked external medium, and the density in both the shell $\rho_{\text {shell }}$ and shocked external medium $\rho_{\text {ext }}$ are given by

$$
\begin{aligned}
& P_{2}\left(r_{2}, t_{2}\right)=f^{2} P_{1}\left(r_{1}, t_{1}\right) \\
& \rho_{\text {shell }, 2}\left(r_{2}, t_{2}\right)=f^{2} \rho_{\text {shell }, 1}\left(r_{1}, t_{1}\right) \\
& \rho_{\text {ext }, 2}\left(r_{2}, t_{2}\right)=f \rho_{\text {ext }, 1}\left(r_{1}, t_{1}\right) .
\end{aligned}
$$

There are several interesting properties of the proposed recipe. First, the magnetizations of both ejecta are the same $\left(\sigma_{0,1}=\right.$ $\sigma_{0,2}$ ), not only at the initial time (which holds from our postulate), but also throughout their evolution, since $\sigma_{2}\left(r_{2}, t_{2}\right) \propto$ $P_{B, 2} / \rho_{\text {shell, } 2} \propto P_{B, 1} / \rho_{\text {shell, } 1} \propto \sigma_{1}\left(r_{1}, t_{1}\right)$. Secondly, $\gamma_{\text {rel }}$ and its time evolution are identical for all models (Fig. 6, upper panel), and thirdly, the time evolution of $\gamma_{\mathrm{FS}}$ is just shifted linearly with $\gamma_{0}$ (Fig. 6, lower panel).

\subsubsection{Invariance of the time of observation}

An important byproduct of the transformations provided by Eqs. (10) and (11) is that the time of observation defined by Eq. (4) is invariant. The only assumption that we make is that the motions of both shells " 1 " and " 2 " are ultrarelativistic, so that the position of the FS can, generally, be written as

$r_{\mathrm{FS}}(t)=c \int_{0}^{t} \mathrm{~d} \tau \beta_{\mathrm{FS}}(\tau) \approx c t-(c / 2) \int_{0}^{t} \mathrm{~d} \tau \gamma_{\mathrm{FS}}^{-2}(\tau)$. 
We first demonstrate the invariance of $t_{\mathrm{obs}}$ for the FS. By inserting Eqs. (13) into (4), we derive for the shell " 2 "

$t_{\mathrm{obs}, \mathrm{FS}, 2}=(c / 2) \Delta_{0,2}^{-1} \int_{0}^{t_{2}} \mathrm{~d} \tau_{2} \gamma_{F S, 2}^{-2}\left(\tau_{2}\right)$

We transform the integral as

$$
\int_{0}^{t_{2}} \mathrm{~d} \tau_{2} \gamma_{\mathrm{FS}, 2}^{-2}\left(\tau_{2}\right)=f^{-8 / 3} \int_{0}^{t_{1}} \mathrm{~d} \tau_{1} \gamma_{\mathrm{FS}, 1}^{-2}\left(\tau_{1}\right),
$$

and insert it into Eq. (14). After transforming $\Delta_{0,2}=f^{-8 / 3} \Delta_{0,1}$, we finally obtain the desired result

$t_{\mathrm{obs}, \mathrm{FS}, 2}=(c / 2) f^{8 / 3} \Delta_{0,1} f^{-8 / 3} \int_{0}^{t_{1}} \mathrm{~d} \tau_{1} \gamma_{\mathrm{FS}, 1}^{-2}\left(\tau_{1}\right)=t_{\mathrm{obs}, \mathrm{FS}, 1}$.

For a point inside the shell "2", that differs from the FS, we have

$t_{\mathrm{obs}, 2}=(c / 2) \Delta_{0,2}^{-1} \int_{0}^{t_{2}} \mathrm{~d} \tau_{2} \gamma_{\mathrm{FS}, 2}^{-2}\left(\tau_{2}\right)-c \Delta_{0,2}^{-1}\left(r_{2}-r_{\mathrm{FS}, 2}\left(t_{2}\right)\right)$.

We can see that the second term on the right hand side is also invariant to the scaling, since $\left(r_{2}-r_{\mathrm{FS}, 2}\left(t_{2}\right)\right)$ and $\Delta_{0,2}$ both scale with $f^{-8 / 3}$. This completes our proof of the invariance of $t_{\mathrm{obs}}$ under the scaling relations of Eqs. (10) and (11).

\subsubsection{Verification by test simulations}

We tested numerically the postulate stated in the previous section for both unmagnetized and magnetized flows, and found that it is correct to within a few percent accuracy. In the following, we present three numerical models that share a common magnetization $\sigma_{0}=1$ and $\xi=1$.1. The remainder of the parameters are: 1) $\gamma_{0,1}=10$ and $\Delta_{0,1}=2.95 \times 10^{15} \mathrm{~cm} ; 2$ ) $\gamma_{0,2}=15$ and $\Delta_{0,2}=1 \times 10^{15} \mathrm{~cm}$; and iii) 3) $\gamma_{0,3}=20$ and $\Delta_{0,3}=2.64 \times 10^{14} \mathrm{~cm}$. We use the scaling relations given in Eqs. (10)-(12) to conform models 1 and 3 to the model 2. In Fig. 5, we show the density, magnetization, and Lorentz factor after applying the scaling laws Eqs. (10)-(12) to the models. We show the scaling after the forward shock of the model 2 has reached a distance $\approx 1.75 \times 10^{17} \mathrm{~cm}$. As we can see, the Lorentz factor and magnetization $\sigma$ scale as expected. The rest-mass density within the shell, and the pressure (not shown here) are also given by Eqs. (12). In Fig. 5, we note that there is a factor of 2 difference between the rescaled results close the RS. The reason for this discrepancy arises from the finite time resolution of our models. To rescale the models we have to use the closest time we have available from the simulation to that requested by the transformation given in Eqs. (11).

\subsection{Extrapolation to $\gamma_{0} \gtrsim 100$ and "bolometric" light curves}

The MHD calculations presented here do not suffice to calculate the expected emission in detail. Such a calculation of the afterglow emission in different observed bands needs additional assumptions related to the, poorly known, shock microphysics. These include, for example, the energy distribution of the accelerated electrons and the generation of magnetic fields in the shock front. Furthermore, one needs to include radiative mechanisms such as synchrotron and inverse Compton and allow for adiabatic losses. This detailed calculation falls beyond the scope of this work. Instead, we apply a simple method to obtain a rough estimate of the bolometric emission expected from the different

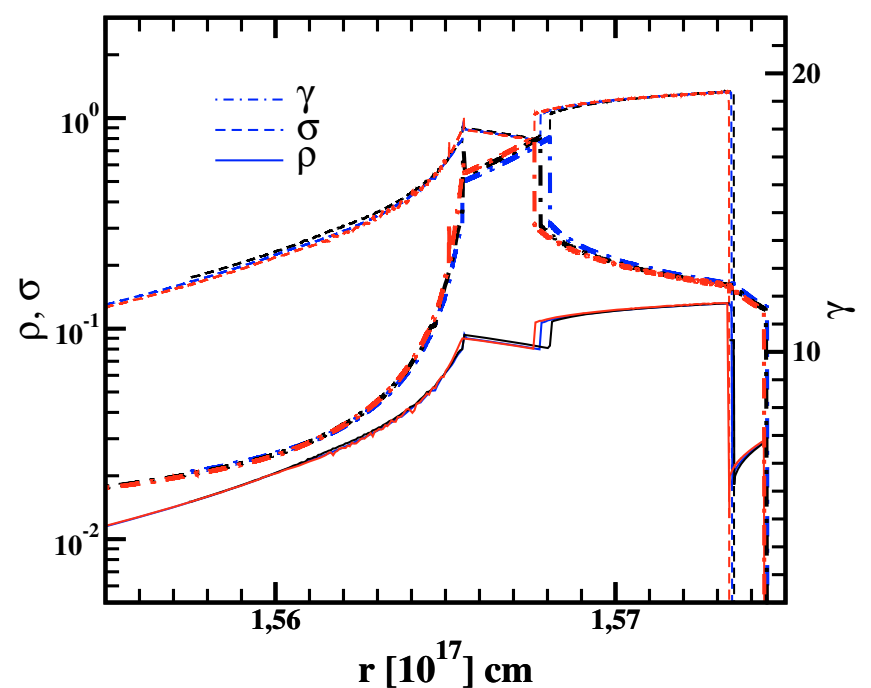

Fig. 5. Results of a test of the rescaling with $\gamma_{0}$. Red, black and blue lines show the results for models with $\gamma_{0}=10,15$, and 20 , respectively. Full, dashed, and dot-dashed lines show the rest-mass density, $\sigma$, and the fluid Lorentz factor, respectively. Models with $\gamma_{0}=10$ and 20 have been rescaled using Eqs. (10)-(12). After rescaling the results, the profiles of all models almost overlap. Only close to RS are there large discrepancies. The reason for them is that we have a finite time resolution and, therefore, we have to rescale our models using the closest time available from the simulation to that requested by the transformation expressed in Eq. (11).

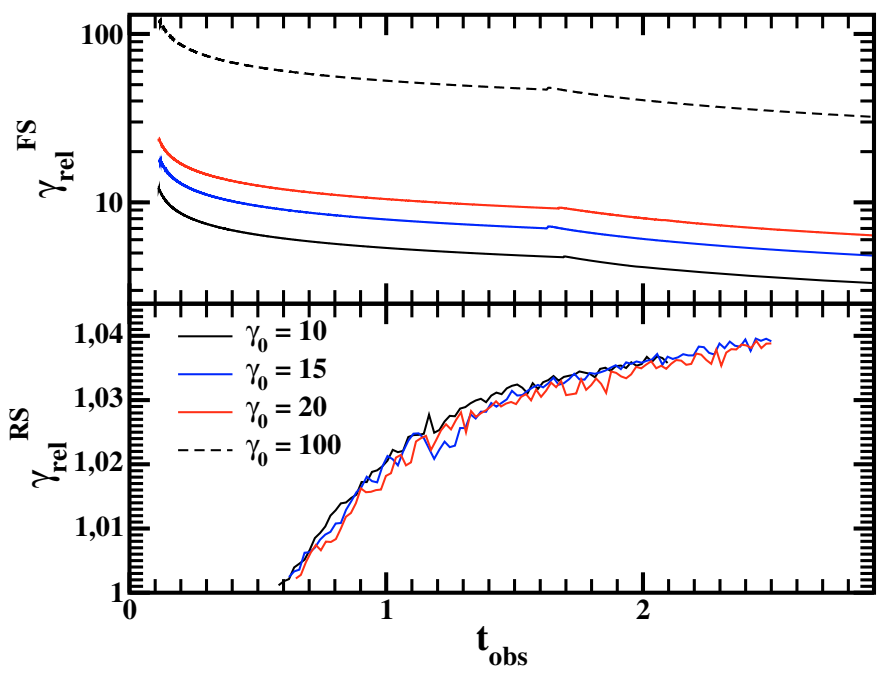

Fig. 6. Similar to Fig. 3, but for the models used in the test of the rescaling hypothesis. Black, blue, and red lines in the upper (lower) panel show the relative Lorentz factor of the fluid at the FS (RS) as a function of the normalized time of observation for models with $\gamma_{0}=10,15$, and 20, respectively. As expected, the relative Lorentz factor at the front shock scales with $\gamma_{0}$, while at the reverse shock they coincide. The dashed line in the upper panel shows the results of a model obtained by rescaling the model with $\gamma_{0}=15$ to $\gamma_{0}=100$.

models. To make predictions about the properties of the afterglow light curves associated with our numerical models, we extrapolate them to the conventionally accepted parameter regime where GRBs occur. To achieve this, we apply the rescaling described in the previous section.

We assume that in both reverse and forward shocks a fraction $\epsilon_{\mathrm{e}} \approx 0.1$ of the dissipated energy ins transferred to high-energy electrons, and that the electrons are in the fast-cooling regime 
(as is usually the case during the initial afterglow phases). The resulting total emission then is given by the rate at which the shocks heat the external medium and the shell.

From the shock conditions at the FS (Sari \& Piran 1995), we derive the thermal energy (in the laboratory frame) dissipated by the FS when it moves from $r$ to $r+\Delta r$ to be

$\Delta E_{\mathrm{th}}(r, t)=16 \pi r^{2} \Delta r \gamma^{2} n_{\mathrm{e}} m_{p} c^{2}$,

where we assume that $\Delta r \ll r$, and that $\gamma$ is approximately constant between $r$ and $r+\Delta r$. We assume the luminosity of the FS to be a fraction of the thermal energy dissipated due to the heating of the external medium by the shell in a time interval $\Delta t=\Delta t_{\mathrm{obs}} \Delta_{0} / c$, where $\Delta t_{\mathrm{obs}}$ is the difference in normalized observational time (Eq. (4)) between the moments in which the FS moves from $r$ to $r+\Delta r$. According to this definition, the bolometric luminosity for the front shock reads

$L_{\mathrm{FS}}:=16 \pi n_{e} m_{p} c^{2} \epsilon_{\mathrm{e}} \gamma^{2} r^{2} \frac{\Delta r}{\Delta t}$.

For the reverse shock, we estimate its luminosity by assuming that a fraction $\epsilon_{\mathrm{e}}$ of the increase in thermal energy inside the shocked shell, as it moves from $r$ to $r+\Delta r$, is radiated by the RS. Thus, we define

$L_{\mathrm{RS}}:=(\Delta t)^{-1} \max \left[0, \epsilon_{\mathrm{e}} \int_{\text {shell } r+\Delta r} 4 \gamma^{2} p-\int_{\text {shell } r} 4 \gamma^{2} p\right]$,

where $\int_{\text {shell } r}$ denotes the integral over the shell volume when FS is at $r$. The integrand $4 \gamma^{2} p$ can easily be derived from the equations of RMHD, assuming an adiabatic index 4/3 (see e.g., Mimica et al. 2007).

The luminosities $L_{\mathrm{FS}}$ and $L_{\mathrm{RS}}$ can be normalized to the initial shell luminosity defined by

$L_{0}:=4 \pi r_{0}^{2} \gamma_{0}^{2} \rho_{\text {shell }} c^{3}$.

In this way, we obtain the dimensionless luminosities $l_{\mathrm{FS}}:=$ $L_{\mathrm{FS}} / L_{0}$ and $l_{\mathrm{RS}}:=L_{\mathrm{RS}} / L_{0}$.

We have checked that the normalized and conveniently scaled light curves for the test models from Sect. 4.4.4 agree to within a few percent of each other. This means that we can use this normalized light curves to predict the bolometric luminosity observed from a shell with, e.g., $\gamma_{0}=100$, and the same value $\xi=1.1$ and 0.5 as our thin and thick shell models, respectively. The light curves computed for the reference models with $\gamma_{0}=15$ and scaled to ejecta of Lorentz factors $\gamma_{0}=100$ (thin shell; Fig. 7) and $\gamma_{0}=300$ (thick model shell; Fig. 8) differ fundamentally between thin and thick magnetized shells. The luminosity of the RS of magnetized thin shells is much smaller than the luminosity of the corresponding RS in the hydrodynamic case (we note that in Fig. 7 the light curve of the magnetized RS is not evident on the scale we are considering). For thick shells, the luminosity of the RS shock is about 10 times smaller than that of the corresponding thick shell with $\sigma_{0}=0$. Hence, the detection of the RS will, in general, be far more difficult if the shell ejecta is magnetized than if it is unmagnetized. Indeed, if the magnetized ejecta is thin, it is likely that the RS will not be detected at all.

\section{Discussion and comparison with previous work}

GRB outflows may be launched by strong fields resulting in a Poynting-flux-dominated wind. In this case, the flow can remain strongly magnetized throughout the acceleration, collimation, and GRB emission phases, all the way to the onset of the

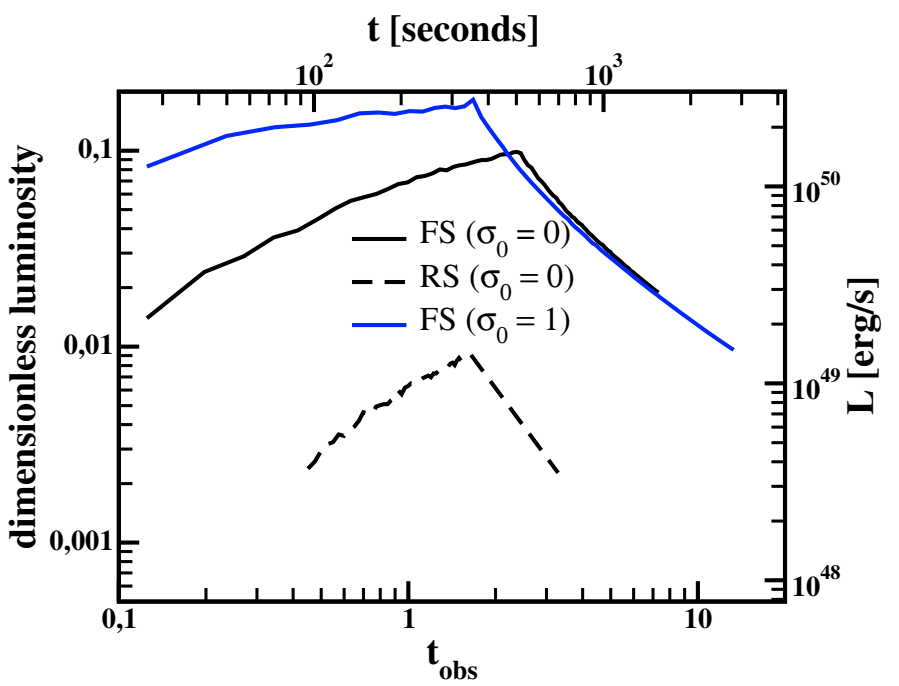

Fig. 7. Light curves for non-magnetized (black) and magnetized (blue line) thin shell models after scaling the $\gamma_{0}=15$ model to $\gamma_{0}=100$. The luminosity of the FS is shown in thick lines, while the black dashed line shows the luminosity of the reverse shock of the unmagnetized shell. The RS of the magnetized shell has a very weak dissipation and its light curve is not shown on the scale of the plot. Left and bottom axes show the dimensionless luminosity $\left(l_{\mathrm{FS}}\right.$ and $\left.l_{\mathrm{RS}}\right)$ and the time of observation, respectively. The displayed luminosity profile can be applied to any other shell with a different $\gamma_{0}$ provided that $\xi=1.1$ (see text). Right and top axes show respectively the luminosity and the normalized time of observation for the particular case $\gamma_{0}=100$.

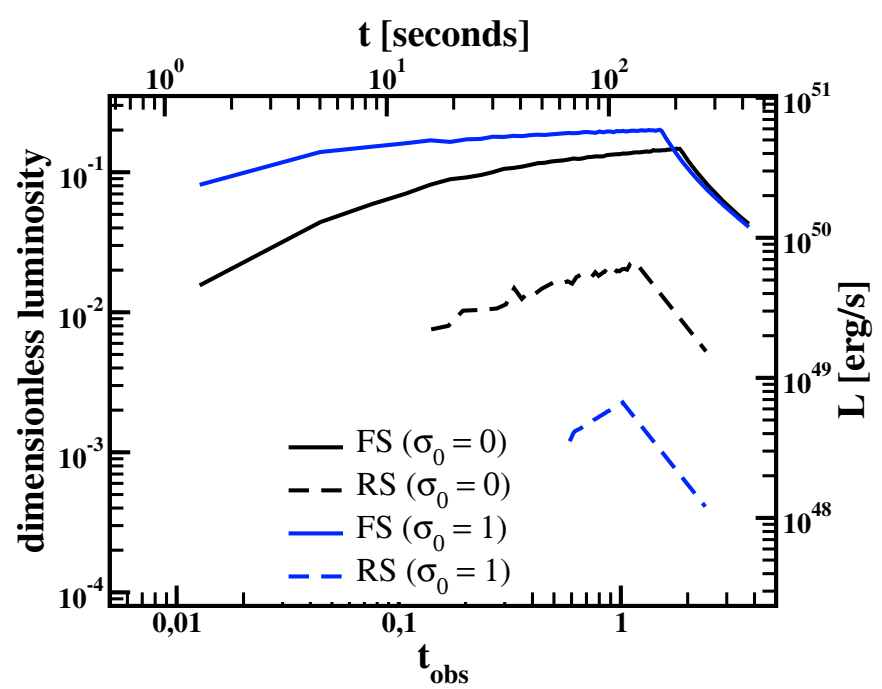

Fig. 8. Same as Fig. 7, but for a thick shell model $(\xi=0.5)$ at $\gamma_{0}=300$. The reverse shock emission from the magnetized thick shell (dashed blue line) is much stronger than in the magnetized thin shell.

afterglow. This is in sharp contrast to expectations for a flow initially dominated by thermal energy (fireball) in which magnetic fields are dynamically unimportant. Magnetization is expected to affect the initial phase of interaction between the GRB ejecta and the external medium. Since early afterglow observations are now possible for many bursts, it has become topical to study the effect of the ejecta magnetization in the early afterglow in more detail.

Here we perform ultra-high resolution 1D RMHD simulations of the interaction between a radially expanding magnetized ejecta and the interstellar medium. We study the complete evolution of an ultrarelativistic, initially-uniform, ejecta shell, all the 
way to a self-similar, asymptotic phase. We explicitly show the resolution of numerical simulations required to resolve appropriately all the discontinuities in the flow, and to be free of numerical artifacts. The main findings of the work are the following:

1. the complete evolution can be characterized in terms of two parameters, namely, the Sari-Piran parameter $\xi$ and the magnetization $\sigma_{0}$. Since both parameters are defined from combinations of basic physical properties of the ejecta (e.g., $\gamma_{0}$, $E$, and $\left.\Delta_{0}\right)$, a single point in the $\left\{\xi, \sigma_{0}\right\}$-plane can be used to probe a variety of equivalent combinations of basic physical parameters. A useful byproduct of this degeneracy is that one can produce numerical models where the shell Lorentz factor is $\gamma_{0}$ is of the order of 10 , and rescale the results to arbitrarily high values $\gamma_{0}$. Certainly, the numerical difficulty of simulations with moderate Lorentz factor (namely, $\gamma_{0} \lesssim 30$ ) is smaller than those with a large one (see Appendix A.1). The later type of simulations are prone to a number of numerical artifacts that hinder an appropriate comprehension of the physics we are dealing with. The method of rescaling our reference numerical models to the conditions expected to occur in GRB afterglows is described in Sect. 4.4;

2. the amounts of energy dissipated in the reverse shock depend strongly on the magnetization of the flow. The reverse shock is weak or absent for ejecta characterized by $\xi \gtrsim 0.5$ (thin shells) and $\sigma_{0} \gtrsim 1$. The emission from the reverse shock is suppressed strongly for $\sigma_{0} \gtrsim 1$, except for high $\gamma_{0} \gtrsim 1000$ flows (or, equivalently, low $\xi$ flows). More moderate values of the magnetization $\sigma \sim 0.1$ can lead to powerful emission, in excess of that expected for $\sigma_{0}=0$ ejecta, since there is both a strong shock and strong magnetic field for efficient synchrotron emission. The last statement assumes that is particle acceleration occurs in magnetized shocks with an efficiency similar to that of unmagnetized shocks;

3. magnetically dominated $\left(\sigma_{0} \gtrsim 1\right)$ ejecta are characterized by an initial rarefaction that originates in the leading radial edge of the shell and results in a Lorentz factor of the front shock $\gamma>\gamma_{0}$. The energy dissipated by the forward shock and the expected emission at the onset of the afterglow are much higher for $\sigma_{0} \gtrsim 1$ ejecta than for the weakly magnetized ones. "Bolometric" light curves were presented in Sect. 4.5;

4. the magnetic energy of the shell is increased by shock compression during the reverse-shock crossing in agreement with the findings of Zhang \& Kobayashi (2005). We have found that the bulk of the magnetic energy is transferred into the shocked external medium on a short timescale (a few to several times the burst duration) for the $\xi=1.1$ and $\xi=0.5$ models that we have simulated. Several lightcrossing times of the initial ejecta width suffice for the evolution of ejecta of $\sigma \sim 1$ to become similar to that of the $\sigma_{0}=0$ simulation. At this stage almost, all the energy has been transferred into the shocked external medium and the forward-shock emission is practically independent of the initial magnetization of the flow. One should observe the onset of the afterglow to identify effects connected to the magnetization.

In comparisson with previous work, Zhang \& Kobayashi (2005) significantly overestimated the reverse-shock emission for $\sigma_{0} \sim$ 1 , mainly because of the assumed higher value of the relative Lorentz factor $\gamma_{\text {rel }}$ (and consequently dissipation) in the reverse shock. The Giannios et al. (2008) analytical curve on the $\left\{\xi, \sigma_{0}\right\}$-plane provided a good approximation of when significant reverse-shock dissipation occurs, concluding that the observed paucity of optical flashes (signatures of reverse-shock emission predicted by the fireball model) may be understood as being due to $\sigma \gtrsim 1$ ejecta is verified by our simulations.

Modelling of the emission associated with the forward and reverse shock can be used to compare the magnetization of the shocked external medium with that of the shocked ejecta (e.g, Zhang et al. 2003). This method was applied to a number of bursts (Fan et al. 2002; Kumar \& Panaitescu 2003; Mundell et al. 2007b; Gomboc et al. 2008). We caution here that such an approach considers hydrodynamical shock conditions and is therefore unreliable when the magnetization of the ejecta is sufficiently high to alter the dynamics of the deceleration. In that a full MHD calculation (such as that presented here) is needed for accurate comparison to observations.

There are aspects of the problem of interaction between magnetized ejecta and the external medium that have not been settled by this work. Although we solved the dynamical evolution of the ejecta, and the strength of the shocks as a function of time, and computed approximate "bolometric" light curves, we did not calculate detailed light curves for different observed bands. For this calculation, additional assumptions would have been required related to the shock microphysics and inclusion of radiative processes such as synchrotron and inverse Compton emission.

Furthermore, we have not explored the high $\xi$ (or Newtonian reverse shock) regime. In this regime, shell-spreading due to the presence of both slower and faster parts within the shell has to be considered. For $\xi \gtrsim 1$, the onset of the afterglow occurs at a later observer time and can be used to infer physical quantities such as the Lorentz factor of the flow $\gamma_{0}$ (Sari \& Piran 1999; Mundell et al. 2007a). The slope of the initial rising part and the peak of the curve depend on the external medium density profile and probably the magnetization of the ejecta. These features are worth investigation in more detail. Finally, since the initial interaction (as seen by the observer) lasts longer, substantial magnetic energy remains in the shell. It is possible that dissipation of this magnetic energy in localized active regions produces late-time flares as proposed by Giannios (2006).

Acknowledgements. The authors thank the referee for his thoughtful comments and suggestions for improvements of this work. P.M. was at the University of Valencia with a European Union Marie Curie Incoming International Fellowship (MEIF-CT-2005-021603). M.A.A. is a Ramón y Cajal Fellow of the Spanish Ministry of Education and Science. P.M. and M.A.A. also acknowledge the partial support from the Spanish Ministry of Education and Science (AYA2007-67626-C03-01, CSD2007-00050). P.M. thanks Jose-Maria Martí and Jose-Maria Ibañez for support and critical discussions. D.G. thanks Henk Spruit for useful discussions. The authors thankfully acknowledge the computer resources, technical expertise and assistance provided by the Barcelona Supercomputing Center - Centro Nacional de Supercomputación.

\section{Appendix A: Numerical method}

We solve equations of RMHD in 1D spherical geometry assuming that the fluid moves only in the radial direction. The magnetic field is purely toroidal magnetic, i.e., the magnetic field is perpendicular to the direction of fluid propagation. The system of RMHD equations is (with the speed of light set to be $c=1$ )

$\frac{\partial \boldsymbol{U}}{\partial t}+\frac{1}{r^{2}} \frac{\partial}{\partial r}\left(r^{2} \boldsymbol{F}\right)=\boldsymbol{S}$

where the vector of unknown or conserved variables is given by

$\boldsymbol{U}=\left(\rho \gamma, \rho h^{*} \gamma^{2} v, \rho h^{*} \gamma^{2}-p^{*}-\rho \gamma, B\right)$ 
The fluxes in Eq. (A.1) are

$\boldsymbol{F}=\left(\rho \gamma v, \rho h^{*} \gamma^{2} v^{2}+p^{*},\left[\rho h^{*} \gamma^{2}-\rho \gamma\right] v, v B\right)$,

and the source terms read

$\boldsymbol{S}=\left(0, \frac{2 p}{r}, 0, \frac{v B}{r}\right)$.

Here $\rho, p, \gamma$, and $B$ are the fluid rest mass density, pressure, Lorentz factor, and magnetic field in the frame of the central engine or laboratory frame. The magnetic field $B$ is measured in Gaussian units. The total pressure is $p^{*}:=p+B^{2} / 2 \gamma^{2}$, and, the specific enthalpy is $h^{*}:=1+\hat{\gamma} p /(\hat{\gamma}-1) \rho+B^{2} / \rho \gamma^{2}$. In our models, the fluid is assumed to be an ideal gas with the adiabatic index $\hat{\gamma}=4 / 3$. We note that we express the components of all three-vectors in the physical, i.e., orthonormal basis.

Romero et al. (2005) discussed the solution to the Riemann problem in for when the magnetic field is perpendicular to the fluid velocity and a Cartesian geometry. They showed that the ratio $B /(\gamma \rho)$ is constant everywhere except across contact discontinuity. An analogous expression in spherical geometry, $\sigma \propto r^{2} \rho$ can be derived by assuming a cold magnetized fluid. In this case, the system of equations given by Eq. (A.1) reduces to three equations. From the continuity and induction equations one can easily derive the desired relation.

We use the relativistic magnetohydrodynamic code MRGENESIS (Mimica et al. 2005, 2007), a high-resolution shock capturing scheme based on GENESIS (Aloy et al. 1999; Leismann et al. 2005). In our code, the fluid is discretized in spherical shells (zones). We use the PPM (Colella \& Woodward 1984) scheme for the spatial interpolation of variables within numerical zones, and a HLLC (Mignone \& Bodo 2006) approximate Riemann solver to compute numerical fluxes across zone boundaries. The time integration is performed using a third-order Runge-Kutta method.

\section{A.1. Numerical resolution}

For the simulation results to be as free as possible of numerical artifacts a sufficiently high resolution is needed. Of particular concern is the minimum number of iterations necessary to resolve the initial evolution of the discontinuity separating the shell from the external medium (that forms a Riemann problem). This information is required since almost all RMHD codes based on approximate Riemann solvers develop initial transient spurious behaviors at the location of the original discontinuity. These spurious behaviors relax with time to the correct physical solution. Therefore, since the problem is self-similar in Cartesian coordinates, almost independently of the initial resolution, our numerical code recovers correctly (i.e., within the accuracy of our method) the physical solution. However, in spherical symmetry the problem is not strictly self-similar. Thus, the break up of an initial discontinuity may lead to the formation of additional discontinuities (inside of the Riemann fan but not directly emerging from the contact discontinuity) at later times. This is precisely what happens in the rarefaction $R_{1}$ in our magnetized models (see Sect. 4.2), where the RS forms. If the formation of the shock happens close to the location of the contact discontinuity, the initial transient artifacts in numerical simulations may pollute the formation of the RS and produce an incorrect numerical solution, where, e.g., the RS does not form. The way to diminish the effect of these initial transients is to increase the numerical resolution around the initial discontinuity.

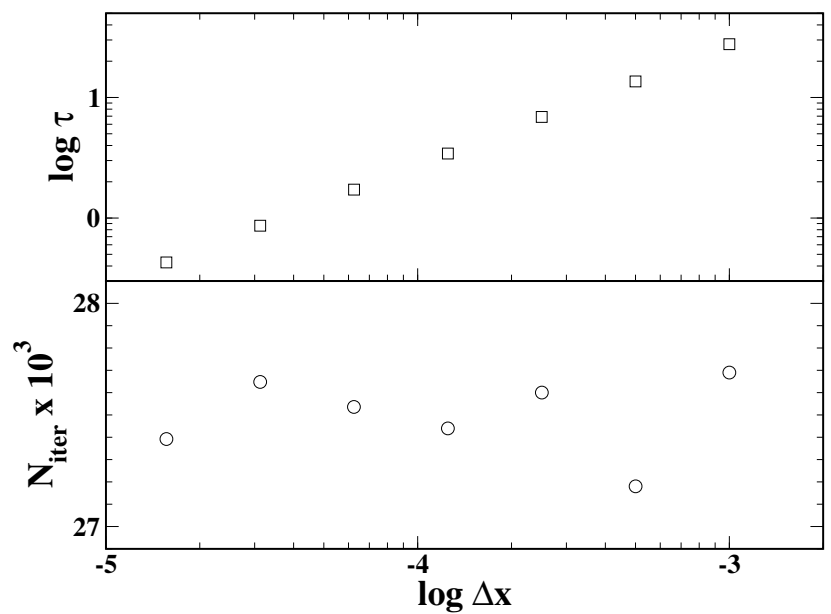

Fig. A.1. Time $\tau$ (upper panel) and the number of iterations $N_{\text {iter }}$ (lower panel) needed to resolve the Riemann problem in planar coordinates as a function of the spatial discretization $\Delta x$.

To eliminate the effects of the spherical geometry on the solution of the Riemann problem, and in view of the fact that our initial shells start at distances of $R_{0} \geq 10^{16} \mathrm{~cm}$, where the local effects of the spherical geometry are practically negligible, we study both exact and numerical solutions of the following Riemann problem in planar coordinates:

- left state: $\rho=1, p=10^{-2}, \gamma_{0}=15$ and $B=15$,

- right state: $\rho=10^{-4}, p=10^{-6}, \gamma=1$ and $B=0$.

The solution to this Riemann problem is self-similar, and consists of a rightward-moving shock separated by a contact discontinuity from a left-moving rarefaction wave. We are interested in the time $\tau$ and number of iterations $N_{\text {iter }}$ a numerical code requires to obtain a correct Lorentz factor (to an accuracy of less than a percent) of the contact discontinuity $\left(\gamma_{\mathrm{CD}}=25.56\right.$ for this particular problem).

Figure A.1 shows the results of the test for seven different simulations whose zone sizes have values $\Delta x=10^{-3}, 5 \times$ $10^{-4}, 2.5 \times 10^{-4}, 1.25 \times 10^{-4}, 6.25 \times 10^{-5}, 3.125 \times 10^{-5}$, and $1.5625 \times 10^{-5}$. By fitting to the data points in the plot, we find $\tau \propto \Delta x^{1.003 \pm 0.002}$. This linear dependence can be seen in the lower panel of Fig. A.1, where we see that $N_{\text {iter }}$ is roughly independent of the resolution (Fig. A.1 lower panel). We find that $\tau \propto \Delta x N_{\text {iter }}$.

Of equal importance is the dependence of $\tau$ and $N_{\text {iter }}$ on the initial Lorentz factor. We modify the Riemann problem by changing $\gamma_{0}$ of the left state and the magnetic field, such that the ratio $B / \gamma_{0}=$ const. For this test, we use the finest resolution $\Delta x=1.5625 \times 10^{-5}$ for all models. Results are shown in Fig. A.2. We find $\tau \propto \gamma_{0}^{1.08 \pm 0.02}$. Due to the constant $\Delta x$, this means that $N_{\text {iter }}$ also depends almost linearly on $\gamma_{0}$. Combining results shown in Fig. A.1 with those of Fig. A.2 and assuming linear dependences of $\tau$ on $\Delta x$ and $\gamma_{0}$, we conclude that the required spatial discretization for Riemann problems similar to those discussed in this paper follows the relation

$\Delta x \propto \tau \gamma_{0}^{-1}$.

We emphasize that the constants implicit to Eq. (A.5) depend on the initial density and pressure ratio, as well as the magnetization of the fluid. The result expressed by Eq. (A.5) can also be interpreted in the following way: at a fixed numerical resolution, if the Lorentz factor of the problem to be solved increases, 


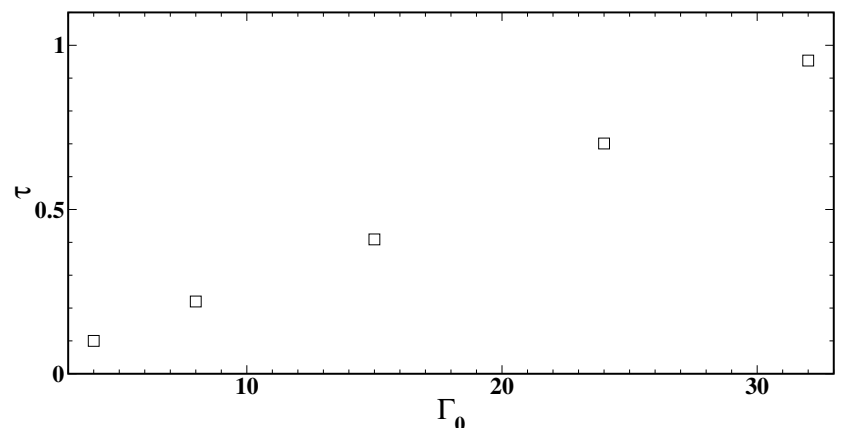

Fig. A.2. Time needed to resolve the Riemann problem in planar coordinates as a function of the initial Lorentz factor $\Gamma_{0}$ for $\Delta x=1.5625 \times 10^{-5}$.

the time needed to relax any initial numerical pathology also increases.

We use Eq. (A.5) to determine the maximum $\Delta x$ (or, conversely, the minimum resolution) needed to complete our simulations such that $\tau$ is far shorter than any of the characteristic hydrodynamic timescales in our models. In articular, we require that $\tau$ is shorter than the time needed to form the RS in the rarefaction fan $R_{1}$.

A reduced density jump between the shell $\rho_{\mathrm{L}}$ and the external medium $\rho_{\mathrm{R}}$ reduces drastically the numerical complexity of the break up of the Riemann problem. Our choice of the density jump $\rho_{\mathrm{L}} / \rho_{\mathrm{R}} \gtrsim 10^{4}$ attempts to reach the large density contrast expected in the conditions found in GRB afterglows (although it is still lower than what an optimal modeling demands). We point out that a much reduced value of $\rho_{\mathrm{L}} / \rho_{R} \sim 10^{2}$ (as considered by Mizuno et al. 2009) could be too low for the conditions in this astrophysical context.

\section{References}

Aloy, M. A., Ibáñez, J. M., Martí, J. M., \& Müller, E. 1999, ApJS, 122, 151 Beskin, V. S., Kuznetsova, I. V., \& Rafikov, R. R. 1998, MNRAS, 299, 341
Blandford, R. D., \& McKee, C. F. 1976, Physics of Fluids, 19, 1130

Colella, P., \& Woodward, P. R. 1984, J. Comp. Phys., 54, 174

Drenkhahn, G., \& Spruit, H. C. 2002, A\&A, 391, 1141

Fan, Y.-Z., Dai, Z.-G., Huang, Y.-F., \& Lu, T. 2002, Chinese J. Astron. Astrophys., 2, 449

Fan, Y. Z., Wei, D. M., \& Wang, C. F. 2004, A\&A, 424, 477

Genet, F., Daigne, F., \& Mochkovitch, R. 2007, MNRAS, 381, 732

Giannios, D. 2006, A\&A, 455, L5

Giannios, D., \& Spruit, H. C. 2006, A\&A, 450, 887

Giannios, D., Mimica, P., \& Aloy, M. A. 2008, A\&A, 478, 747

Gomboc, A., Kobayashi, S., Guidorzi, C., et al. 2008 [arXiv : 0804 . 1727]

Goodman, J. 1986, ApJ, 308, L47

Granot, J., Miller, M., Piran, T., Suen, W. M., \& Hughes, P. A. 2001, in Gammaray Bursts in the Afterglow Era, ed. E. Costa, F. Frontera, \& J. Hjorth, 312

Kennel, C. F., \& Coroniti, F. V. 1984, ApJ, 283, 694

Kobayashi, S., Piran, T., \& Sari, R. 1999, ApJ, 513, 669

Kumar, P., \& Panaitescu, A. 2003, MNRAS, 346, 905

Leismann, T., Antón, L., Aloy, M. A., et al. 2005, A\&A, 436, 503

Li, Z.-Y., Chiueh, T., \& Begelman, M. C. 1992, ApJ, 394, 459

Lyutikov, M. 2005 [arXiv:astro-ph/0503505]

Lyutikov, M. 2006, New J. Phys., 8, 119

Lyutikov, M., \& Blandford, R. 2003, ArXiv Astrophysics e-prints

Meliani, Z., Keppens, R., Casse, F., \& Giannios, D. 2007, MNRAS, 376, 1189

Meszaros, P., \& Rees, M. J. 1997, ApJ, 482, L29

Michel, F. C. 1969, ApJ, 158, 727

Mignone, A., \& Bodo, G. 2006, MNRAS, 368, 1040

Mimica, P., Aloy, M. A., Müller, E., \& Brinkmann, W. 2005, A\&A, 441, 103

Mimica, P., Aloy, M. A., \& Müller, E. 2007, A\&A, 466, 93

Mizuno, Y., Zhang, B., Giacomazzo, B., et al. 2009, ApJ, 690, L47

Mundell, C. G., Melandri, A., Guidorzi, C., et al. 2007a, ApJ, 660, 489

Mundell, C. G., Steele, I. A., Smith, R. J., et al. 2007b, Science, 315, 1822

Paczynski, B. 1986, ApJ, 308, L43

Rees, M. J., \& Gunn, J. E. 1974, MNRAS, 167, 1

Rees, M. J., \& Meszaros, P. 1992, MNRAS, 258, 41P

Rhoads, J. E. 1999, ApJ, 525, 737

Romero, R., Martí, J. M., Pons, J. A., Ibáñez, J. M., \& Miralles, J. A. 2005, J. Fluid Mechanics, 544, 323

Sari, R., \& Piran, T. 1995, ApJ, 455, L143

Sari, R., \& Piran, T. 1999, A\&AS, 138, 537

Thompson, C. 1994, MNRAS, 270, 480

Usov, V. V. 1992, Nature, 357, 472

Vlahakis, N., \& Königl, A. 2003, ApJ, 596, 1080

Zhang, B., \& Kobayashi, S. 2005, ApJ, 628, 315

Zhang, B., Kobayashi, S., \& Mészáros, P. 2003, ApJ, 595, 950 